\title{
Review
}

Fetal Diagnosis and Therapy
Fetal Diagn Ther 2015;38:81-85

DOI: $10.1159 / 000382015$
Received: November 6, 2014

Accepted after revision: March 27, 2015

Published online: May 13, 2015

\section{Fetal Therapy for Isolated Aqueductal Stenosis}

\author{
Stephen P. Emery ${ }^{a}$ Stephanie Greene ${ }^{b}$ William Allen Hogge ${ }^{a}$ \\ Departments of a Obstetrics, Gynecology and Reproductive Sciences and ${ }^{\mathrm{b}}$ Neurological Surgery, \\ The University of Pittsburgh School of Medicine, Pittsburgh, Pa., USA
}

\section{Key Words}

Isolated aqueductal stenosis · Fetal hydrocephalus · Fetal surgery · Ventriculoamniotic shunt

\begin{abstract}
Aqueductal stenosis (AS) is a form of noncommunicating hydrocephalus, which causes increased intracranial pressure secondary to obstruction of the aqueduct of Sylvius. Relief of intracranial pressure in the fetus by ventriculoamniotic shunting may diminish or even prevent permanent neurologic injury. Shunting was attempted in the 1980s but was abandoned due to technical difficulties. Given the advances in prenatal diagnosis and fetal intervention over the last 3 decades, we believe that an evidence-based reevaluation of the option is timely and appropriate. The aim of this review article is to discuss the clinical significance of the diagnosis of AS, current management strategies, current diagnostic capabilities, new shunt technology, and barriers to progress. Finally, we will advance a research agenda that will provide evidence-based management recommendations.
\end{abstract}

(c) 2015 S. Karger AG, Basel
() 2015 S. Karger AG, Basel

$1015-3837 / 15 / 0382-0081 \$ 39.50 / 0$

\section{Background}

Severe fetal central nervous system (CNS) ventriculomegaly is a relatively common prenatal diagnosis, occurring at a rate of $0.3-0.5$ per 1,000 deliveries $[1,2]$. The majority of cases of severe ventriculomegaly are secondary to a primary process such as encephalocele, DandyWalker malformation, or spina bifida [3]. Neurologic outcomes are generally dismal [2]. Aqueductal stenosis (AS), however, is a form of severe fetal CNS ventriculomegaly that may have a better neurologic outcome, as the ventriculomegaly is not secondary to an underlying malformation, deformation, or destructive process [4]. AS leads to fetal noncommunicating hydrocephalus by obstruction of the flow of cerebrospinal fluid (CSF) at the level of the aqueduct of Sylvius between the third and fourth ventricles, the narrowest portion of the ventricular system. The causes and timing of the obstruction are unknown. Although not evident in the first trimester [5], typical sonographic findings can be observed by 20 weeks' gestation [6]. The pathogenesis of AS is that of supratentorial intraventricular hypertension [7], resulting in a pressure gradient between the third and fourth ventricles and transmission of the normal pulsatility of the CSF to the cortical mantle, leading to progressive ventricular di-

\section{KARGER 125}

E-Mail karger@karger.com www.karger.com/fdt
Stephen P. Emery, MD

Magee-Womens Hospital of the University of Pittsburgh Medical Center 300 Halket Street

Pittsburgh, PA 15213 (USA)

E-Mail semery@mail.magee.edu 
lation. Neurological sequelae include upgaze palsy, blindness, abnormal hypothalamic function, intellectual decline, and spasticity. Findings in newborns include failure to thrive, developmental delay, apnea, bradycardia, and a setting sun sign. The natural history of isolated AS remains difficult to define, as many cases are terminated or are analyzed along with other forms of severe ventriculomegaly [8]. Levitsky et al. [9], however, reported on a series of 30 patients with isolated AS confirmed on postnatal imaging. The mortality rate was $40 \%$ (12 of 30 patients). Eight (27\%) died within the first 3 months of life. Ninety-five percent of those who survived the neonatal period underwent ventricular shunting. Only 10\% (3 of 30) were developmentally normal after long-term followup. This dismal prognosis has led to the concept of prenatal intervention with a ventriculoamniotic shunt to hopefully relieve intraventricular hypertension, normalize cerebral blood flow and ventricular size, and prevent progressive neurologic injury.

\section{Fetal Intervention for Severe Hydrocephalus}

Because of poor postnatal neurologic outcomes, efforts were made in the 1980s to intervene prenatally in order to bypass the obstructed aqueduct by means of serial cephalocentesis [10] and ventriculoamniotic shunting $[11,12]$. Ventriculoperitoneal shunting in newborns dramatically improved survival [13]. It seemed reasonable that fetal shunting would improve neurologic outcomes by minimizing hydrocephalus-related damage during early brain development. Efforts in humans were hampered, however, by poor subject selection and by technical difficulties [14]. A de facto moratorium was placed on shunting for fetal hydrocephalus in 1986 [14]. Little progress has been made in the management of fetal hydrocephalus since.

The concept of prenatal decompression of congenital noncommunicating hydrocephalus is appealing on many levels. Based on our current understanding of the pathogenesis of AS, obstruction of CSF at the aqueduct of Sylvius results in increased intracranial pressure or intraventricular hypertension. This hypertension results in decreased cerebral blood flow, regional ischemia, and an altered microenvironment of the periventricular neurons [7]. Progressive ventricular dilation secondary to the CSF pulsatility being absorbed by the cortex leads to axonal shear and gliosis [15]. Cortical dysgenesis may result from the early development of ventriculomegaly. Cerebral blood flow and ventriculomegaly have both been shown to be readily normalized with shunting in laboratory animals [16], with more recovery associated with shorter durations of hydrocephalus. Bypassing the intraventricular obstruction prenatally may allow for more normal brain development and can allow the pregnancy to progress to term with ventricular shunting in the newborn period [17]. This approach may be preferable to current management strategies of early delivery and newborn shunting or expectant management to term. A drawback of early delivery is a consequent poor surgical candidate due to prematurity and a higher incidence of shunt-related complications [18]. Disadvantages to expectant management include ongoing cortical dysgenesis as well as current and future obstetric implications of the probable need for a vertical ('classical') uterine incision due to often massive craniomegaly [19]. Given the poor neurologic prognosis of the newborn, this added maternal risk seems to tilt the risk-benefit assessment in an unfavorable, albeit unavoidable, direction.

In utero treatment of congenital AS meets most of the criteria for in utero intervention set out by the International Fetal Medicine and Surgery Society (IFMSS) in 1982 [20]. Animal models for fetal hydrocephalus were developed in the early 1980s [7, 21]. Early shunt placement in an animal model has been associated with improved axon transport [22] and normalization of myelin production [23]. Initial results in humans were promising $[11,12]$. An international registry of procedures was initiated under the auspices of the IFMSS. Unfortunately, enthusiasm for the concept exceeded the ability to accurately diagnose isolated AS, and fetuses with lesions other than AS (holoprosencephaly, Dandy-Walker malformation, porencephalic cyst, Arnold-Chiari malformation, and arthrogryposis) were shunted [14]. These lesions would be unlikely to benefit from in utero treatment of hydrocephalus. Importantly, patients with a diagnosis of $\mathrm{X}$-linked hydrocephalus were not excluded from the analysis. The inclusion of a heterogeneous population inherently obscures the results in any small subset of the group. Nonetheless, a moratorium was put in place, and prenatal ventriculoamniotic shunting as well as medical progress regarding the prenatal diagnosis of severe hydrocephalus essentially ceased.

It is important to recognize that the only patients within the registry who demonstrated benefit from prenatal shunting were those with the diagnosis of AS on postnatal evaluation. Forty-two percent of these were normal on follow-up. The other $58 \%$ were mildly, moderately, or severely neurologically impaired [14]. This likely represents an improvement in the natural history of AS, though it
Emery/Greene/Hogge 
may not have been appreciated at the time. Given the advances in prenatal diagnosis and intervention, it has been recently suggested that the concept of prenatal shunting should be revisited $[24,25]$.

\section{Prenatal Diagnosis of AS}

In the intervening decades since the initial attempts at in utero shunting, there have been significant advances in prenatal diagnosis such as high-resolution and 3-dimensional ultrasound, MRI, and sophisticated genetic testing that can more accurately detect potential candidates for in utero intervention. Fetal isolated AS has a specific sonographic appearance of symmetric severe CNS ventriculomegaly, thinning of the brain parenchyma both anteriorly and posteriorly, a dangling choroid plexus, a dilated third ventricle, and a preserved posterior fossa. The head circumference is usually normal or enlarged. MRI findings are loss of the subarachnoid space and no evidence of a cerebral aqueduct distal to the third ventricle [26]. The overall appearance is that of increased intraventricular pressure. This is a different sonographic appearance than what is observed in developmental disorders in which CSF occupies the space that the brain does not (colpocephaly), or that of destructive lesions that are usually asymmetric and have a normal-to-small head circumference (porencephaly). The observation of cerebellar hypoplasia, for instance, points to other concerns such as neuronal migration disorders that are causing narrowing or obstruction of the aqueduct. Destructive processes such as infection or hemorrhage can clog the aqueduct with debris, resulting in the sonographic appearance of AS. For these reasons, the final diagnosis of isolated AS should be based on serial imaging studies as well as on genetic (L1CAM mutation or X-linked hydrocephalus), infectious (rubella, CMV, and toxoplasmosis) and hematologic (antiplatelet antibody) testing.

In a recent retrospective review of 110 fetuses with severe ventriculomegaly, we were able to diagnose isolated AS with $100 \%$ accuracy [6]. Additionally, we were able to differentiate isolated AS from AS with other subtle CNS findings (cerebellar hypoplasia, tectal plate mass, or hemorrhage) with similar accuracy. The median gestational age at ultrasound diagnosis was 23 weeks +3 days, with a range of 19 weeks +6 days to 33 weeks +0 days. This wide range reflects the referral nature of our patient population. The median age at MRI was 33 weeks +3 days, with a range of 28 weeks +4 days to 34 weeks +0 days, which reflects our propensity to perform MRIs in the mid-third

Fetal Therapy for Isolated Aqueductal Stenosis trimester. The median difference in gestational age by head circumference was +16 days (range -2 to 30 ). The median lateral ventricular diameter was $20 \mathrm{~mm}$ (severe ventriculomegaly $\geq 15 \mathrm{~mm}$ ) [27]. This detection rate is in contrast to a reported $20-30 \%$ false positive-rate in earlier studies $[8,14]$.

Prenatal diagnosis of AS will need to take place at an early gestational age if successful intervention is to be entertained. This can in all likelihood be achieved given our current experience with 2- and 3-dimensional ultrasound, combined with increasing experience with MRI in the mid trimester. Finally, advances in genetic testing such as microarray and whole exome sequencing can aid in proper patient selection. A fetus with apparent isolated AS but with a duplication or deletion on microarray may not be a good candidate for prenatal intervention, especially if that specific rearrangement has a known phenotype beyond that expected for isolated AS, or if a gene defect associated with severe neurologic outcomes is present (for example L1CAM mutation or X-linked hydrocephalus) [28].

Successful intervention may also depend on our ability to accurately predict the proper timing of intervention; to intervene before permanent cortical damage has occurred is essential. Serial ultrasounds can likely detect the rate of cortical thinning and calvarial distention. Color Doppler interrogation may be useful to detect altered intracranial blood flow (for example middle cerebral artery flow velocity) as a result of increased intracranial pressure. An animal model is underway that is intended to provide information about the appropriate timing of intervention.

\section{New Shunt Technology}

There were several technical problems with the shunts used in the 1980s. Placed by ultrasound guidance similar to vesicoamniotic or thoracoamniotic shunts (thereby avoiding the maternal morbidity associated with laparotomy and hysterotomy), they had a tendency to obstruct and to migrate [7]. A single published series of patients who underwent ventriculoamniotic shunt placement by hysterotomy documented a lower shunt failure rate [29]. However, the need for hysterotomy negatively impacts upon the overall risk-benefit assessment. Newer shunt technology may overcome earlier shortcomings. Requirements for a ventriculoamniotic shunt are straightforward: ultrasound-guided percutaneous insertion, an anchoring mechanism to prevent migration, a one-way 
valve to prevent amniotic fluid reflux, and sufficient compliance to avoid cerebral injury. A shunt with these performance characteristics can likely be engineered with modern technology and materials already approved for use in humans. A prototype has been constructed and is undergoing in vitro testing at the University of Pittsburgh Swanson School of Engineering.

\section{Barriers to Progress and Research Agenda}

Because of the experience with ventriculoamniotic shunting in the 1980s and the existing moratorium, a scientifically rigorous and evidence-based reassessment will be required before shunting in human fetuses can be resumed. Criteria for fetal surgery as proposed by the IFMSS include: (a) accurate diagnosis and staging is possible, with exclusion of associated anomalies; (b) the natural history of the disease, including prognosis, is known; (c) current postnatal therapy is ineffective; (d) feasibility and effect are demonstrated in an animal model, and (e) interventions are performed in specialized multidisciplinary fetal treatment centers with appropriate oversight and informed consent $[20,30]$. In a retrospective analysis, we have demonstrated that not only can isolated AS be diagnosed prenatally, it can be discriminated from AS with other subtle CNS anomalies [6]. The next phase is a prospective assessment of natural history as well as our ability to prenatally diagnose isolated AS through the North American Fetal Therapy Network (NAFTNet). NAFTNet is a consortium of 30 medical institutions in the US and Canada with established expertise in fetal surgery and other forms of multidisciplinary care for complex fetal disorders. It includes representations from maternal-fetal medicine, pediatric surgery, pediatric cardiology, neonatology, biostatistics, and bioethics. This multidisciplinary approach provides scientific and bioethical oversight, transparency, and a comprehensive, evidence-based perspective. A protocol has been proposed to prospectively assess the performance characteristics of prenatal ultrasound and MRI to timely diagnose fetuses that may benefit from in utero intervention for isolated AS. These fetuses/newborns will be followed for a period of time and will represent a cohort of contemporary postnatal management (ventriculoperitoneal shunt, endoscopic third ventriculostomy) of AS with which a future prenatal management cohort (ventriculoamniotic shunting) can be compared. Longitudinal assessment of this cohort throughout pregnancy and early childhood can improve our understanding of the natural history of the disease.
Fetoscopic fenestration of the floor of the third ventricle or endoscopic third ventriculostomy can be explored as a means of avoiding the need for an indwelling shunt prenatally and may provide sufficient decompression until definitive treatment is achieved in the newborn period. Endoscopic third ventriculostomy in infancy is successful only in 25\% [31], and the risk of injury to adjacent structures (the pituitary gland, optic chiasm, or basilar artery) may preclude this as a therapeutic option. The size of the endoscopy equipment necessary to safely perform the procedure in a fetus may also be prohibitive, and the puncture site will need a watertight seal. Nonetheless, this option should be investigated in an animal model.

Because of the rarity of isolated AS ( $<4,000$ cases/year in the US), a mechanical shunt device will likely be considered a humanitarian use device. This has implications for US Food and Drug Administration (FDA) regulation and approval. There will likely be challenges in the manufacturing of the shunt as well. However, once the prototype shunt is available, the next step will be feasibility testing in an animal model. We plan to use an induced hydrocephalus sheep model that was extensively described in the 1980s [32]. Once feasibility and safety are demonstrated in the animal model, and the natural history of isolated AS is better defined through a prospective cohort followed through NAFTNet, the device will be ready for human trials, which will be conducted through select NAFTNet centers.

\section{Summary}

Fetal severe hydrocephalus is associated with a poor neurologic prognosis. AS causes cerebral injury through intraventricular hypertension. The natural history of AS may be improved upon by fetal ventriculoamniotic shunting. Mechanical shunting was attempted in the 1980 s but was abandoned because of the inability to accurately identify suitable candidates. Advances in prenatal diagnosis and fetal therapy in the intervening decades may allow for the identification of a patient population amenable to in utero intervention, namely fetuses with isolated AS diagnosed in the mid trimester. We are proposing an evidence-based reassessment of ventriculoamniotic shunting through NAFTNet. Only NAFTNet, through scientifically rigorous, transparent, multidisciplinary, and collaborative research, is in the position to overturn the moratorium. Indeed, this collaborative research model may prove to be the best approach for the future study of rare but significant fetal diseases. 


\section{References}

1 Wiswell TE, et al: Major congenital neurologic malformations. A 17-year survey. Am J Dis Child 1990;144:61-67.

$>2$ Hannon T, et al: Epidemiology, natural history, progression, and postnatal outcome of severe fetal ventriculomegaly. Obstet Gynecol 2012;120:1345-1353.

$\checkmark 3$ Nyberg DA, et al: Fetal hydrocephalus: sonographic detection and clinical significance of associated anomalies. Radiology 1987;163: 187-191.

4 Drugan A, et al: The natural history of prenatally diagnosed cerebral ventriculomegaly. JAMA 1989;261:1785-1788.

5 Senat MV, et al: Prenatal diagnosis of hydrocephalus-stenosis of the aqueduct of Sylvius by ultrasound in the first trimester of pregnancy. Report of two cases. Prenat Diagn 2001;21:1129-1132.

$\checkmark 6$ Emery SP, et al: Accuracy of prenatal diagnosis of isolated aqueductal stenosis. Prenat Diagn 2015;35:319-324.

7 Glick PL, et al: Correction of congenital hydrocephalus in utero II: efficacy of in utero shunting. J Pediatr Surg 1984;19:870-881.

8 Hudgins RJ, et al: Natural history of fetal ventriculomegaly. Pediatrics 1988;82:692-697.

$>9$ Levitsky DB, et al: Fetal aqueductal stenosis diagnosed sonographically: how grave is the prognosis? AJR Am J Roentgenol 1995;164: 725-730.

10 Birnholz JC, Frigoletto FD: Antenatal treatment of hydrocephalus. N Engl J Med 1981; 304:1021-1023.

11 Frigoletto FD Jr, Birnholz JC, Greene MF: Antenatal treatment of hydrocephalus by ventriculoamniotic shunting. JAMA 1982;248: 2496-2497.
12 Clewell WH, et al: A surgical approach to the treatment of fetal hydrocephalus. N Engl J Med 1982;306:1320-1325.

13 Ransohoff J, Shulman K, Fishman RA: Hydrocephalus: a review of etiology and treatment. J Pediatr 1960;56:399-411.

14 Manning FA, Harrison MR, Rodeck C: Catheter shunts for fetal hydronephrosis and hydrocephalus. Report of the International Fetal Surgery Registry. N Engl J Med 1986;315: 336-340.

5 Del Bigio MR: Neuropathological changes caused by hydrocephalus. Acta Neuropathol 1993;85:573-585.

16 Del Bigio MR, Bruni JE: Changes in periventricular vasculature of rabbit brain following induction of hydrocephalus and after shunting. J Neurosurg 1988;69:115-120.

-17 Del Bigio MR, Bruni JE: Periventricular pathology in hydrocephalic rabbits before and after shunting. Acta Neuropathol 1988;77: 186-195.

18 McGirt MJ, et al: Cerebrospinal fluid shunt survival and etiology of failures: a seven-year institutional experience. Pediatr Neurosurg 2002;36:248-255.

19 Patterson LS, O’Connell CM, Baskett TF: Maternal and perinatal morbidity associated with classic and inverted T cesarean incisions. Obstet Gynecol 2002;100:633-637.

20 Harrison MR, et al: Fetal treatment 1982. N Engl J Med 1982;307:1651-1652.

21 Michejda M, Hodgen GD: In utero diagnosis and treatment of non-human primate fetal skeletal anomalies. I. Hydrocephalus. JAMA 1981;246:1093-1097.
22 Eskandari R, et al: Effects of hydrocephalus and ventriculoperitoneal shunt therapy on afferent and efferent connections in the feline sensorimotor cortex. J Neurosurg 2004;101(2 suppl):196-210.

23 Del Bigio MR, Kanfer JN, Zhang YW: Myelination delay in the cerebral white matter of immature rats with kaolin-induced hydrocephalus is reversible. J Neuropathol Exp Neurol 1997;56:1053-1066.

24 Saadai P, et al: Fetal neurosurgery: current state of the art. Future Neurol 2011;6:165171 .

25 von Koch CS, et al: In utero surgery for hydrocephalus. Childs Nerv Syst 2003; 19:574-586.

26 Logan A, McLean M: Aqueductal stenosis; in Woodward PJ (ed): Diagnostic Imaging: Obstetrics, ed 2. Salt Lake City, Amirsys Inc., 2011.

27 Gaglioti P, et al: Fetal cerebral ventriculomegaly: outcome in 176 cases. Ultrasound Obstet Gynecol 2005;25:372-377.

28 Weller S, Gartner J: Genetic and clinical aspects of X-linked hydrocephalus (L1 disease): mutations in the L1CAM gene. Hum Mutat 2001;18:1-12.

29 Davis GH: Fetal hydrocephalus. Clin Perinatol 2003;30:531-539.

30 Deprest JA, et al: The making of fetal surgery. Prenat Diagn 2010;30:653-667.

31 Kulkarni AV, et al: Endoscopic third ventriculostomy in the treatment of childhood hydrocephalus. J Pediatr 2009;155:254-259.e1.

32 Nakayama DK, et al: Correction of congenital hydrocephalus in utero I. The model: intracisternal kaolin produces hydrocephalus in fetal lambs and rhesus monkeys. J Pediatr Surg 1983;18:331-338. 